\title{
Importancia del Habitar en el Pensamiento Arquitectónico
}

\author{
Importance of Living in Architectural Thought \\ Importância de viver no pensamento arquitetônico
}

\author{
Joely Ariagny Sulbarán Sandoval. Ph.D en Arquitectura. \\ joely.sulbaran@fad.luz.edu.ve \\ (iD) https://orcid.org/0000-0002-9752-0648 \\ Universidad del Zulia. República Bolivariana de Venezuela. \\ Rafael Humberto Rangel Rojas. M.Sc en Gerencia de Proyectos. \\ leafarangel.arquitectura@gmail.com \\ (iD) https://orcid.org/0000-0002-3522-8481 \\ Universidad del Zulia. República Bolivariana de Venezuela.
}

Recibido: Agosto 30 de 2018 Aceptado: Noviembre 11 de 2018 Publicado: Diciembre de 2018

\section{RESUMEN}

Esta investigación pretende desglosar la complejidad del pensamiento arquitectónico desde su origen en el habitar, a través del empleo de la hermenéutica como medio de interpretación, basado en las teorías de autores como Ramírez, Saldarriaga, Iglesia, Heidegger, Doberti, Cuervo, Aisa, entre otros, quienes se dedicaron a reflexionar la puesta en práctica de la habitabilidad de la arquitectura, concluyendo que el saber arquitectónico exige ser abordado en su complejidad, lo que implica una integralidad de las diferentes áreas del conocimiento para el manejo conceptual. Igualmente, la importancia que tiene la interpretación de la relación espacio-ser humano para el desarrollo de la vida

Palabras clave: habitar; habitabilidad; Arquitectura; pensamiento complejo; reflexión.

\section{ABSTRACT}

This research aims to break down the complexity of architectural thought from its origin in the habitat, through the use of hermeneutics as a means of interpretation, based on the theories of authors such as Ramírez, Saldarriaga, Iglesia, Heidegger, Doberti, Cuervo, Aisa, among others, who dedicated themselves to reflect on the implementation of the habitability of architecture, concluding that architectural knowledge demands to be addressed in its complexity, which implies an integrality of the different areas of knowledge for conceptual management. Equally, the importance of the interpretation of the space-human being relationship, for the development of life.

Keywords: inhabit; habitability; Architecture; complex thinking; reflection; space-human being relationship

\section{RESUMO}

Esta pesquisa tem como objetivo quebrar a complexidade do pensamento arquitetônico desde sua origem no habitat, através do uso da hermenêutica como um meio de interpretação, baseado nas teorias de autores como Ramírez, Saldarriaga, Iglesia, Heidegger, Doberti, Cuervo, Aisa, entre outros, que se dedicaram a refletir sobre a implementação da habitabilidade da arquitetura, concluindo que o conhecimento arquitetônico exige ser abordado em sua complexidade, o que implica uma integralidade das diferentes áreas do conhecimento para a gestão conceitual. Da mesma forma, a importância da interpretação da relação espaço-humano, para o desenvolvimento da vida.

Palavras-chave: habitar; habitabilidade; Arquitetura; pensamento complexo; reflexão.

Como citar (APA)

Sulbarán Sandoval, J., \& Rangel Rojas, R. (2018). Importancia del Habitar en el Pensamiento Arquitectónico. Procesos Urbanos 5:26-33. DOI: https://doi. org/10.21892/2422085X.405 


\section{Introducción}

Con el objeto de desglosar esa complejidad del pensamiento arquitectónico $y$, principalmente, para tratar de determinar cuál es la raíz de muchas de las posturas que hoy en día merecen mayor reflexión y puesta en práctica en el tema de la habitabilidad de la arquitectura, se hace mención de una serie de autores que han estudiado los temas de arquitectura y habitabilidad.

Donde cada uno de estos autores, desde su época, busca mejorar la arquitectura a través de su visión crítica de la misma. Cabe destacar que existen infinidades de autores y teorías referentes a habitabilidad y arquitectura. No obstante, en este estudio se resaltan los más relevantes o los que comparten una postura hacia la reflexión de la arquitectura desde un punto de vista ético de la habitabilidad, cuyos enunciados refieren que la arquitectura es el medio para la habitabilidad de la especie humana y un fin en sí mismo.

Se puede decir que la arquitectura $y$, por consiguiente, la habitabilidad son complejas por su naturaleza, por provenir del ingenio del hombre para solventar sus propias necesidades y las de otros seres humanos. Y, como es una estructura compleja, por comprender en ellas reglamentos que permiten mejorar la calidad de vida del ser humano habitador, se hace necesario nombrar al padre de la complejidad, "Edgar Morin", con el objeto de tener un mayor conocimiento del contexto de dicho tema utilizando el método de la hermenéutica para complementar la interpretación de dichos conceptos y, posteriormente, encaminarlos en el área de la arquitectura y su relación con la habitabilidad.

Afirma Morin (2004) que, la palabra complejidad proviene de complexus, que significa "lo que esta conjuntamente plegado". Igualmente, afirma que en la complejidad ya no existe una relación causa-efecto, tal como era concebido el conocimiento en la ciencia clásica. Este describe la complejidad como una relación tridimensional, donde existe una causa, un efecto $y$, a raíz de ese efecto, se produce otra causa, y así sucesivamente. Donde hay una relación interminable de fenómenos y eventos naturales, sociales y humanos, lo que nos lleva a decir que todo está relacionado de algún modo, siendo una especie de circulo propiamente dicho, el circulo hermenéutico, dónde todas las "partes" de un "todo" se relacionan entre sí para formar el todo y este todo, compuesto por las partes, es mayor que la suma de ellas.

Adicionalmente, Morin (2009) agrega que es necesario separar las partes del todo para ser estudiadas de forma aislada, tal como en la ciencia clásica. No obstante, afirma que la complejidad considera necesaria la unión de las partes anteriormente separadas, para estudiar el todo, ya contando con los datos recogidos al observar e interpretar dichas partes, generando un conocimiento del comportamiento del "todo". De hecho, este conocimiento no es compatible con el modelo especular de la ciencia clásica (realidad hecha, acabada y plenamente externa y objetiva, donde el aparato cognoscitivo es un espejo que la refleja dentro de sí), por el hecho de que la complejidad considera que la verdad es relativa y cambiante. No comparte la ingenuidad del paradigma positivista, donde se consideraba que se podía conocer de forma absoluta la realidad del objeto de estudio.

Por lo antes expuesto, podemos decir que la arquitectura es una estructura compleja que tiene como fin el habitar, y que habitar en sí es parte de la arquitectura. Asimismo, existen muchos factores que es necesario tomar en cuenta al momento de diseñar, construir y habitar. Bauman (2006) nos permite percibir una red compleja de acontecimientos que afectan a la sociedad y su cultura, creando conciencia de la realidad, y la forma en que se están desenvolviendo muchos acontecimientos de la vida actual, que vienen gestándose desde la modernidad. La vida líquida y la modernidad líquida - de las que habla Bauman (2006) - están estrechamente ligadas.

Asimismo, la sociedad moderna líquida es aquella en que las condiciones de actuación de sus miembros cambian antes de que las formas de actuar se consoliden en unos hábitos y en unas rutinas determinadas. La vida liquida no puede mantener su forma ni su rumbo durante mucho tiempo. En la sociedad moderna líquida, la industria de la eliminación de residuos pasa a ocupar los puestos de mando de la economía de la vida líquida. La supervivencia de dicha sociedad y el bienestar de sus miembros dependen de la rapidez con la que los productos quedan relegados a meros desperdicios, y de la velocidad y la eficiencia con la que estos se eliminan.

Esta cultura de liquidez se aprecia no solo en la vida de los seres humanos, en su forma de habitar, su cultura, sus gustos y sus percepciones de los espacios, de la ciudad, de la arquitectura. Todo cambia muy rápidamente de dirección. Esto también es a causa de la creciente globalización y de la hiperevolución de la tecnología. Esto acrecienta la velocidad con la que las cosas llegan a su obsolescencia y, por consiguiente, el comportamiento de las personas cambia con la misma velocidad por el hecho de no querer quedar "obsoletos". Ese querer estar al día con todas las informaciones a nivel mundial, las últimas tendencias entre otras, y la capacidad de estar en contacto con personas al otro extremo del mundo con una llamada. Se acortan las distancias, se incrementa la velocidad del ritmo de vida.

Por su parte, Morin (2008) define la cultura como la fuente generadora/regeneradora de la complejidad de las sociedades humanas. Integra a los individuos en la complejidad social y cotidiana el desarrollo de su complejidad individual. De tal forma, la cultura es la vía de transmisión de caracteres adquiridos por las sociedades. La sociedad humana se autoproduce, se autoorganiza, se autoperpetúa, se autorregula a partir de reglas, saberes, mitos, normas prohibiciones de una cultura, que operan la incorporación social de los individuos.

Los autores mencionados anteriormente de alguna manera se complementan y apoyan la teoría de que la situación actual de la realidad es compleja, se vive en una incertidumbre tras otra, lo que lleva en muchos casos a un fluir con las tendencias y gobiernos. No hay nada que dure ni mucho menos el hombre tiene la necesidad de que dure; la sociedad está acostumbrada a asumir riesgos, a la rapidez y fluidez del momento, al consumismo, a vivir al límite del día a día. No es posible construir planes a largo plazo y que sean eficaces al momento de terminar. Estamos en constante cambio y, muchas veces, no se tiene el tiempo de reflexionar cada uno de los acontecimientos.

La cultura -como bien dice Morin (2008) - tiene la capacidad de autoregenerarse, autoproducirse, autoorganizarse $y$, adicionalmente, es capaz de mutar, transformarse, fluir, descomponerse y volverse a crear, en esta época, a una velocidad impresionante. Forma parte del pensamiento complejo, donde la realidad se convierte en un bucle infinito y que, además, se entreteje de multidiversidad. 


\section{Orígenes del conocimiento de la habitabilidad}

\section{Habitar}

"Habitar es el rasgo fundamental del ser, conforme al cual los mortales son".

"Nuestro modo de ser en el mundo consiste en habitarlo" Martin Heidegger (1956).

Según estas citas, los seres humanos no tenemos otra opción en el mundo que habitar, y es una cualidad del ser y del estar propia del ser humano. Asimismo, afirma Heidegger, que el habitar seria en todo caso el fin que persigue todo construir, "(...) la palabra habitar señala hacia algo ineludible para todos los seres humanos (...). No existe ninguna persona que no habite y no hay momento alguno que no lo haga: habitamos y habitamos siempre" (Doberti, 1999, p. 1-5).

Por su parte, Saldarriaga (2006) expresa: habitar es ocupar un territorio y vivir en él. Vivir quiere decir, según él, exactamente, que se dispone de un lugar especialmente destinado para tal fin, donde se puede permanecer, sentirse seguro, y hacer todas las cosas cotidianas que forman parte de la vida del ser. Habitar es afirmar la presencia de vida en el territorio, es disponer de espacios y edificaciones construidas específicamente para alojar a los seres humanos y solventar sus necesidades, es hacer su vida más fácil y placentera. Para el ser humano habitar significa supervivencia, la conciencia de la muerte, el paso del tiempo, que la vida misma le es propia, dándole así una defensa contra la muerte, ya que la habitación es un espacio ligado propiamente con la angustia existencial del ser humano y el habitar en un espacio seguro, capaz de dar alivio a éste.

Por lo tanto, se puede decir que nuestro modo de ser en el mundo consiste en habitarlo, y en todo momento de nuestra existencia habitamos, el lugar puede ser cualquiera, ya sea en una vivienda, un edificio, la escuela, la ciudad, entre otros, dónde nos encontremos siempre estamos en constante habitar, se puede afirmar que está dentro de nuestra naturaleza y proviene de nuestro origen como especie.

Esa ocupación del territorio o del lugar es propia del habitar $y$, por consiguiente, de los seres vivos, ya que el cuerpo físico exige propiamente ocupar un espacio. El ser humano, en especial, es la única especie que reflexiona sobre este habitar $y$, en medio de ese proceso, es capaz de cambiar y transformar el entorno, siempre tratando de mejorar sus condiciones para que el entorno le permita vivir con mayor seguridad y calidad de vida. Por el mismo hecho es que el ser humano siempre busca que su habitar en el mundo este enfocado a una vida buena, donde pueda desarrollarse tanto económica, social y hasta emocionalmente, donde sus futuras generaciones en el mayor de los casos tenga mejores comodidades.

Añade Doberti (1993, p. 1), la cercanía, la cotidianidad y familiaridad del habitar tiene como consecuencia que no se reconozcan sus incógnitas, sus opacidades, su compleja y velada estructuración. Indica Cuervo (2009) que el problema de la incomprensión del territorio de habitar y la escasa relación con los asuntos epistemológicos de diseño, reside en el olvido o desconocimiento del ser.

Por esta misma razón refiere: "...los estudios más cercanos a la arquitectura, se han preocupado mucho por el entorno como objeto, marcando diferencias en tiempos y lugares distintos, más que por el habitante como sujeto de la acción habitar". (Iglesia, S/F(Mimeo) p.5).
No se puede negar que, en las condiciones de la vida actual, como se habló anteriormente, casi no hay un tiempo para reflexionar acerca de la misma, lo cual influye en el habitar y por consiguiente en la percepción de los arquitectos. Iglesia (2010) refiere que los estudios de arquitectura se encargan de la obra arquitectónica como pieza de arte. Lo podemos observar en los libros de arquitectura de cualquier época, dónde se habla casi siempre de la descripción del edificio, acabados, materiales y hasta conceptos, pero casi nunca de cómo es la vida del habitante. Otra razón, como afirma Doberti, es el asumir ciertas posturas porque el habitar se hace tan cotidiano que pasa desapercibido en muchos casos. Esto no exonera de su reflexión. Al contrario, es un recordatorio de la necesidad de enfrentarse a un reto más complejo, como es la reflexión del ser humano en la arquitectura, como su principal beneficiado e invitando al arquitecto a ser un profesional integrado.

Según Doberti (1993), a diferencia de las otras especies, el ser humano - por tener la capacidad de hablar y pensar- es capaz de construir y habitar. A pesar de que otras especies pueden crear refugios, el ser humano es la única especie capaz de poder reflexionar acerca de tal acción, por lo tanto, se puede hablar de habitar. Adicionalmente, asegura Doberti, que habitamos de forma muy variada, podemos afirmar que es por la misma complejidad del ser humano y esa libertad que, a pesar de ser parte de una sociedad en constante evolución, es libre de establecer su propia forma de habitar. Complementando lo anterior, Villagrán afirma "(...) la meta del arquitecto y del estudiante no puede ser en ningún caso la obra representada, sino la obra viva, habitada y ambientada". (Ramírez, 2012, p. 1).

Además, afirma Ramírez (2012), la arquitectura es el arte de construir espacialidades en la que el hombre integral desenvuelve parte de su existencia colectiva. Dentro de su complejidad del ser humano, se puede llevar a que esa existencia colectiva forma parte de su ser como parte de la sociedad.

La sociedad, como parte fundamental del crecimiento y del desenvolvimiento pleno de la vida del ser humano, es parte de ese entorno habitable, la cual va a ser la encargada de colocar los valores culturales, los conocimientos, la historia, la ideología, los valores morales, socioeconómicos, políticos entre otros, que formarán ese contexto habitable del ser.

Saldarriaga (1981) afirma que este habitar se da entre el ser humano y la naturaleza, entre el equilibrio y su reciprocidad, que son elementos que se contemplan o por lo menos debería ser así. Para que el habitar exista, debe tener cierto proceso de transformación, procesos que cada a día son más elaborados y complejos, gracias a nuestras sociedades que están en constante transformación y que - según él- toma el camino de una transformación destructiva, en lugar de una constructiva.

El habitar es algo tan abstracto como la vida misma; es dinámico, cambiante y siempre es capaz de modificarse en el tiempo y en el espacio. El habitar pertenece a la complejidad propia del ser humano (individuo-sociedad-especie), y esta se ve enriquecida con los factores que la constituyen y le dan forma, como es el caso del lugar habitado, las costumbres y tradiciones (cultura), todos esos aspectos sociales que se suman a la personalidad del ser y en la parte individual dónde el hombre es capaz de discernir qué puede y qué no puede hacer, que está dentro de sus posibilidades, la moral, la ética en sus acciones, los valores sociales y los infundidos por la familia, todo compone esa red infinita de tejidos que forman el ser y por consiguiente su habitar. 
Entonces, señalando o determinando los aspectos que componen esa complejidad de habitar, cabe preguntarse: ¿Puede el arquitecto, formado para responder formalmente a las necesidades de los seres humanos a través de técnicas de construcción, responder adecuadamente a los requerimientos de un usuario? Planteado de esa forma se ameritaría el empleo de más de una rama de la ciencia. Se hace necesario un equipo multidisciplinar (psicólogos, antropólogos, biólogos, ambientalistas, políticos, economistas, arquitectos, ingenieros, entre otros), dónde se pueda hacer un estudio más profundo de la realidad $y$, por consiguiente, una respuesta a las necesidades más adaptada a la realidad del ser. $\mathrm{O}$, como dijera Ramírez, una mejor interpretación del habitar.

Como complemento, Benavides y Hurtado (2015) plantean que la habitabilidad es la esencia de la arquitectura y en la actualidad debería ser un ejercicio interdisciplinario: urbanismo, diseño industrial, ingeniería, sociología, filosofía, biología, ciencias políticas, que logren integralidad con miras a proporcionar calidad de vida y bienestar a los habitantes que logren llevar el concepto de habitabilidad y arquitectura a otras escalas, como la de la ciudad. Además, no se trata de un concepto estático que no cambie con los modos de vida de las sociedades y la situación geográfica, sino, más bien, un concepto dinámico, flexible, capaz de transformarse para poder adaptarse a los requerimientos.

Según Saravia (2004), habitar es la huella de la vida, es dejar huella de la existencia humana. Añade además Illich "...la habitación, como huella de la vida (nunca acabada, nunca completamente planificada), florece y decae al compás de los esplendores y fracasos de sus habitantes" (Saravia. 2004, p. 1).

Agrega Heidegger (1956), que las edificaciones auténticas acuñan al morar su esencia, solo cuando podemos pensar en habitar podemos edificar (...) Pensar y edificar son indispensables para morar (Ver Figura 1).

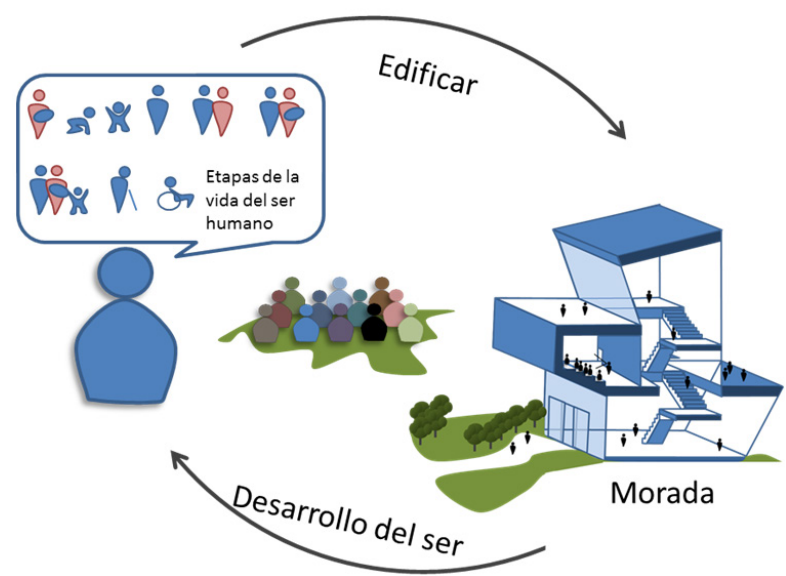

Figura 1. Habitar según Heidegger.

Fuente: Sulbarán, 2017

En el mismo tono, Cuervo (2009) considera el habitar como una acción necesaria para el desarrollo social, cultural e individual del hombre, y la arquitectura como una posibilidad en la cual se desarrolla dicha acción (habitar). Por lo tanto, la arquitectura y el habitar no pueden ser dos asuntos contrarios, sino relacionados e interdependientes el uno del otro. Conforme a lo antes expuesto, Ramírez (2012) afirma que la habitabilidad implica ineludiblemente la relación entre el espacio habitable y el hombre habitador; adicionalmente, refiere Ramírez (2012, p. 9), los arquitectos no inventamos conceptos, no depende de nuestra "creatividad" sino de la correcta interpretación del habitar. Mientras mejor sea esa interpretación del habitar, será más trascendental la arquitectura y permitirá más apropiación de los habitantes. Por el contrario, si la interpretación es mala, o sencillamente la edificación carece de ella, no podría ser arquitectura, ya que perdería esa razón primordial o sentido de la arquitectura.

Según la teoría de la arquitectura de Villagrán "...la forma arquitectónica maneja un espacio construido doble: el edificado que es el construido por material y el habitable que es el que ocupa el cuerpo humano". (Ramírez, 2012, p.7)

Entonces, lo habitable comprende la relación entre los espacios arquitectónicos con sus diferentes condiciones y el ser humano habitador. Dichos espacios construidos representan los medios y el ser humano se refiere a la satisfacción de las necesidades, y ambos dan como fin la arquitectura.

Asimismo, asegurá Saligon, citado por Iglesia, que el habitar consta de un sentido (S/F(Mimeo), p. 32): "(..)si el habitar tiene sentido, es porque le permite al ser humano tener acceso a una serie de intencionalidades como espacio-tiempo esencial de proyectos, de futurizaciones, de transformaciones, de apropiaciones y de identificaciones, que hacen que la vida este ligada a la manera de habitar".

Se puede inferir que la personalidad, la cultura, el contexto económico, social, los valores morales y éticos, hasta la parte afectiva, psicológica y bilógica, se ven reflejados en su forma variada de habitar. Esa particularidad del ser es la que le permite de una u otra forma el enraizarse, emocionarse, recordar y apropiarse de los espacios en los que se ve reflejado. $\mathrm{O}$, en su defecto, odiar, condenar y olvidar, si no son de su agrado.

Apoyando lo anteriormente expuesto, Heidegger y NorbergSchulz acuñaron el concepto de espacio existencial para referirse al espacio habitado o habitable: "(...) el entorno habitado o habitable adquiría carácter al ser identificado (usado) por el hombre, al ser significativo; esto lo llamo espacio existencial". (Iglesia, 2010, p. 18)

Por su parte, Martínez (2014) afirma que la apropiación exige en todo momento la necesidad y el deseo de hacer. Respecto a la noción de apropiación espacial se advierten ciertamente interpretaciones muy variadas. No siempre son reconocibles en la óptica fijada por el autor, que pivota de manera significativa en torno al análisis de las contradicciones de la sociedad y las posibilidades de una transformación.

Por consiguiente, se hace necesario ese pensar en las necesidades de cada uno de los habitadores de los espacios y cómo, de manera técnica, es posible solventar dichas necesidades aplicando el ingenio del arquitecto en el proceso, a pesar de que, como dice Ramírez (2012), no se trata de inventar una nueva forma de habitar se trata de interpretar correctamente esos patrones de habitabilidad que tienen los moradores de los espacios, para que ellos puedan apropiarse e identificarse con estos y la relación entre espacio- hombre sea lo más estrecha posible.

A eso mismo nos refiere Alexander (1981), el cual considera que cada ciudad y todo edificio están formados por ciertas entidades llamada lenguaje de patrones, que surgen de ciertos procesos combinatorios. El lenguaje de patrones está orientado a originar una estructura viviente, y crear, de esa forma, una arquitectura 
que se conecte con la sensibilidad humana. Según Alexander, se trata de un proceso a través del cual el orden de un edificio o de una ciudad surge directamente de la naturaleza interna de la gente, los animales, las plantas y las materias que los componen, lo que permite que la vida interior del individuo, de una sociedad, o una ciudad florezca abiertamente en libertad.

Estos lugares poseen la cualidad "viviente" en la medida de la habitabilidad que posean, generando los patrones de acontecimientos, es decir, el carácter del lugar viene dado por los acontecimientos que allí ocurran. Todos estos patrones de acontecimientos van a depender siempre de la cultura de los individuos. Se puede decir que esta es quien influye dentro de los comportamientos y, por consiguiente, en la arquitectura (Ver Figura 2).

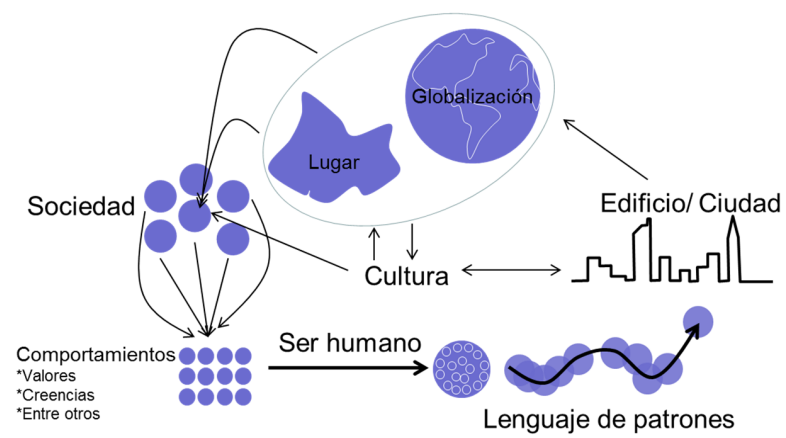

Figura 2. Influencia de la cultura en la arquitectura.

Fuente: Sulbarán, 2015.

Por su parte, Leff, citado por Correa (2007) considera que habitar, "es el lugar en el que se construye y se define la territorialidad de una cultura, la especialidad de una sociedad y de una civilización, donde se constituyen los sujetos sociales que diseñan el espacio geográfico apropiándoselo, habitándolo con sus significaciones y prácticas, con sus sentidos y sensibilidades, con sus gustos y goces".

Illich citado por Saravia (2004, p. 2-6) refiere que comprende el habitar territorio: "(...) habitar el territorio es marcarlo... reconocerlo y recorrerlo... convivirlo... compartirlo y construirlo; es hacer, manipular, utilizando herramientas ya sean manejables o manipulables... es entender y comprender el territorio y sus hechos culturales e históricos, tradiciones... no hay mejor forma de entenderlo que a través de hábitos de conocimiento".

Entonces, si consideramos el ser humano (satisfacer a sus necesidades, y considerar su forma propia de habitar) como fin del quehacer arquitectónico, es necesario analizar lo que vuelve a un espacio habitable, con el firme propósito de mejorar la calidad de vida del habitador.

Por su parte, López (2011) reflexiona sobre el texto de Heidegger y afirma que la verdadera necesidad de habitar existe en el hecho de que los mortales buscamos siempre de nuevo la esencia de habitar, deben aprender a habitar en el mundo, por lo tanto hay que incorporar en el habitar elementos y valores del medio que puedan proporcionar una mayor riqueza cualitativa de fruiciones.

Se puede afirmar que la esencia del habitar siempre va a ser relacionada a la existencia humana y al edificar como fin último de este. Pero, a pesar de eso, el habitar comprende un proceso complejo que contempla los factores que afectan la vida del ser y que enriquecen su vida, así como la experiencia. Los saberes que ese ser alcance a lo largo de su vida, se convierten en los insumos de edificar, ya que, a partir de ellos, es que se puede llegar a una correcta interpretación de las necesidades de este. Esa reflexión del modo de habitar generará espacios más humanos y que posean una significación para el habitante, generando así un sentido de apropiación del espacio y, como dijera Alexander (1981), un espacio viviente (Ver Figura 3).

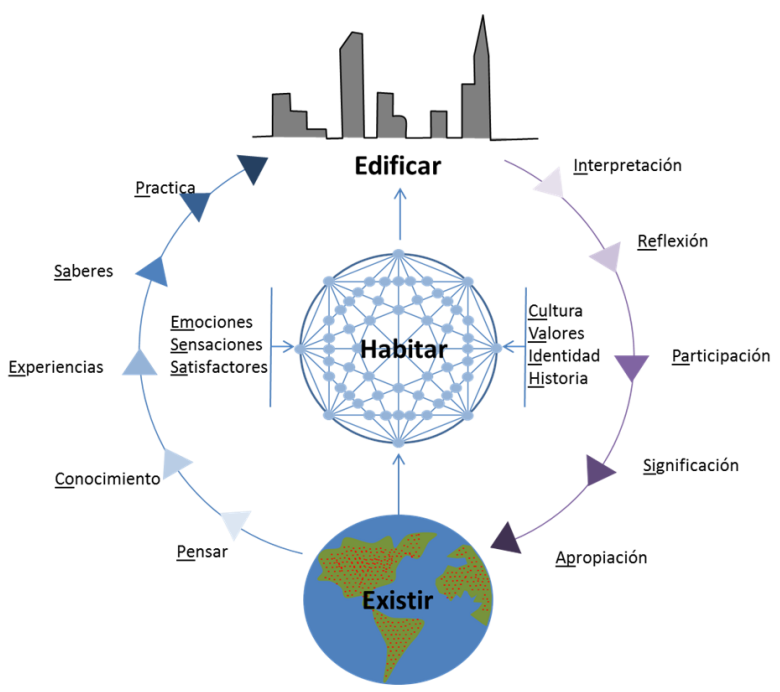

Figura 3. Esencia del habitar.

Fuente: Sulbarán, 2017.

Anteriormente, se explica que los espacios deben llenar condiciones que les permitan a estos cumplir las exigencias del ser humano que los habita. Por tal razón, se hace necesario el estudio, en primer lugar, de ese ser humano integral (individuosociedad-especie) que habita los espacios, que se apropia de ellos a medida que los vive en su cotidianidad, para después plantear espacios edificados (arquitectura). Y, además, afirma Yori (1999) que en estos espacios puedan detenerse y enraizarse en el que dejen huellas.

Por su parte, Cuervo (2009), habitar siempre se piensa o es el deber ser, bajo la mirada funcional del espacio. $Y$ este, en relación con el ser humano, permanece como un instrumento para mejorar la vida. Agrega Cuervo, el habitar le permite al habitador tres características propias: la realización (por el hecho de permanecer en un lugar); el habitar como significación (construirse una memoria y deseos); y el habitar como una expresión (el proyectar y habitar es una expresión en el mundo).

Asimismo, Aisa (2012) expone que el hombre con sus manos y su inteligencia no especializada transforma el mundo: lo habita. La inteligencia y sus creaciones culturales ayudan al hombre a crearse su mundo. Sin duda, la capacidad de habitar ha de verse íntimamente ligada a la espacial condición de la realidad humana.

Como ya antes se había dicho, la realidad para la ciencia clásica era representada como un hecho especifico, donde se creía plenamente tener el conocimiento objetivo de esta, lo cual, con el correr de los años, se determinó que la realidad es relativa, coyuntural y llena de incertidumbre. Tal como se habló, estamos en un constante fluir, donde la realidad se transforma con mucha facilidad. No existe certeza absoluta de ella, y que el ser humano se dedica a vivirla sin mayor complicación, dejándose llevar en muchas ocasiones por los acontecimientos. 
Según lo anteriormente mencionado, con respecto al termino de habitar, podemos afirmar que habitar es, entonces, una característica propia del ser en el mundo; que todo habitar representa una complejidad, por tratarse de un ser humano pensante y capaz de reflexionar, libre y que se expresa a través del derecho propio. Habitar, también se dijo que está directamente relacionado con un medio físico dónde se construye la vida y el cual, mayormente, esta manipulado por el mismo ser humano. La arquitectura, por su parte responde a las necesidades del ser habitador; debe presentar ciertas características o condiciones, lo que se conoce como "habitabilidad".

\section{Habitabilidad}

Según Mues (2011), la habitabilidad está determinada por la relación y adecuación entre el ser humano y su entorno. Se refiere a la forma en que cada una de las escalas territoriales es evaluada, según su capacidad de satisfacer las necesidades humanas.

Según la Real Academia Española, "(...) la habitabilidad se define como: cualidad de lo habitable, y en particular la que con arreglo a determinadas normas legales, tiene un local o una vivienda". (Molero, 2014, p. 62).

Por su parte, Arai expone que: "El complejo concepto de la habitabilidad comprende tanto al habitante como a la habitación, así como a la recíproca relación que se establece entre ellos (...)". (Ramírez, 2012, p. 30).

Con respecto a lo anterior, añade Mercado (1995), la habitabilidad es el grado en que una edificación se ajusta a las necesidades y expectativas de los moradores. Por consiguiente, la habitabilidad de ese edificio irá de acuerdo con el grado en que las expectativas de cada habitador se cumplan conforme a sus necesidades y al estilo de vida que tengan en el mismo. Se puede inferir que la habitabilidad representa la relación de dos componentes fundamentales para la vida del ser en el mundo: uno muy complejo -como es el caso del habitante con todas sus necesidades y expectativas- y el otro es el edificio o espacio construido -el cual debe cumplir con ciertas condiciones que vienen dadas por el lugar la cultura, todo el cumplimiento de normas y expectativas del habitante-(Ver Figura 4).

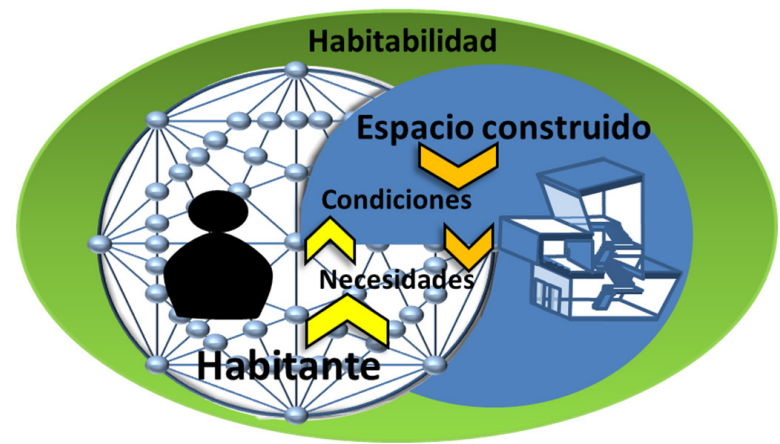

Figura 4. Habitabilidad.

Fuente: Autor, 2017.

Agrega Mercado (1995), que la habitabilidad viene definida por muchos factores físicos y psicológicos que hacen parte de la cotidianidad. Asimismo, la relación del hombre y el medio, ya sea natural o construida, para lograr la armonía hombre-ambiente, y el concientizar a una responsabilidad en su influencia sobre él, no es algo sencillo, ni pertenece a una sola corriente del pensamiento. No solo la define la arquitectura, sino también otras ramas de la ciencia, tales como la psicología, la filosofía, la tecnología, las ciencias sociales, las naturales, entre otras.
Otra definición de habitabilidad, complementaria a la definición arrojada por Mercado, es la definición de habitabilidad sugerida por Michelsen y Read: "...es el resultado de elecciones acerca de cómo las personas asignan sus recuerdos, tanto económicos como simbólicos, esfuerzos gustos y preferencias". (Benavides y Hurtado, 2015, p. 44).

Según ellos, cuando se habla de habitabilidad se habla también de lo que es la calidad de vida, definiendo esta como la percepción que el sujeto tiene de sí mismo, y esto representa un indicador de bienestar personal.

López (2010) agrega que, en el diseño arquitectónico, cuando la habitabilidad se ha estudiado y garantizado, comienza el juego de lo formal y se lleva de significado y capacidad expresiva, mientras mantiene dichas garantías. Si en el juego se pierde la relación con la habitabilidad, lo formal se convierte en accesorio, superficial, frívolo e insustancial.

Alcántara y Gomez afirman que, "(...)la habitabilidad es la condición esencial de la arquitectura y todo programa arquitectónico considera en forma relevante la ventilación, la iluminación y extensión visual, como aspectos determinantes de la habitabilidad". (Benavides y Hurtado, 2015, p. 45).

Según Arcas, Pages y Casal (2007), la habitabilidad debería vincular la persona y sus necesidades. Por lo tanto, reconocer que existe una amplia variedad de situaciones en función de la edad, sexo, condición social y cultural, religión, entorno, entre otros, que exigen respuestas concretas y derivadas de esta especialidad. Lo que lleva a una habitabilidad interpretable, principalmente a partir de las demandas sociales de cobijo, se articula desde el consumo de utilidades, desde la satisfacción de necesidades y no, como hasta ahora, viene siendo el resultado de la producción de más espacios.

La habitabilidad debería ser diversificada, adaptable y proporcionar respuestas adecuadas a la variedad de modos de vida y estructuras de convivencia presentes en la actualidad y a su evolución en el tiempo.

Según Delgadillo (2013), el objeto de estudio del hábitat, es en sí la propia complejización del hábitat. Se aborda desde dos potencialidades del hábitat-habitabilidad, viendo a ambos como dos conceptos diferentes, pero complementarios.

Cabe destacar que Saldarriaga (2006, p. 6) explica que, "(...) la transformación de habitar en hábitat no es un puro juego de lenguaje. Aun cuando hábitat es un término de reciente aparición en el lenguaje del urbanismo y de la arquitectura, y es además prestado de la ecología, ya es de uso común y se refiere a todo aquello que tiene que ver con los asentamientos humanos en la superficie terrestre. Hábitat es, en un sentido más amplio, todo el territorio habitado por la humanidad".

Agrega Mena (2011) que los criterios para analizar y evaluar la habitabilidad no pueden ser universales, claro que estos varían de acuerdo con la persona, la cultura, el lugar, el clima, entre otros. La habitabilidad se entiende como una meta del bienestar del ser humano.

La habitabilidad es un estado generado a partir de las cualidades satisfactorias de necesidades y aspiraciones de los residentes, relación permanente entre el ser humano y su entorno.

Por último, se presenta un gráfico resumen de los tres términos, habitar, hábitat y habitabilidad (Ver Figura 5). 


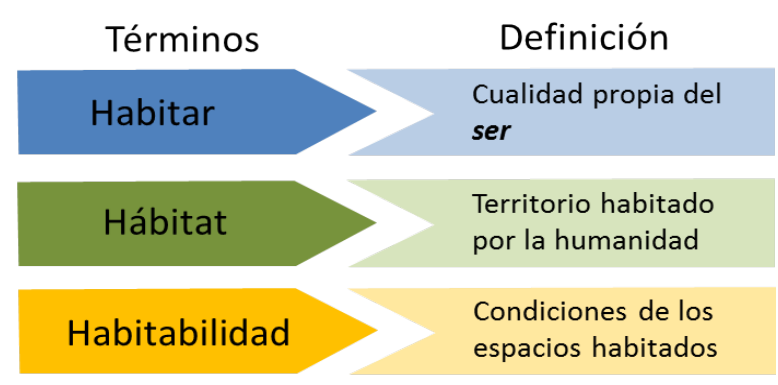

Figura 5. Términos de referencia y síntesis de sus definiciones. Fuente: Sulbarán 2017

\section{Consideraciones finales}

Primeramente, es necesario recalcar que la arquitectura la vamos a concebir desde una visión ética de la habitabilidad. Por consiguiente, no toda construcción es arquitectura, lo que nos lleva a que esta debe cumplir siempre con su propósito de mejorar las condiciones de vida de los seres humanos y a afectar lo menos posible el lugar de emplazamiento, brindando un equilibrio entre hombre-espacio físico construido-ambiente.

Para fines de interpretar la complejidad que representa el habitar y poder llegar una respuesta arquitectónica se hace necesario, primero, el cambio de mentalidad del arquitecto, basado en la ciencia clásica, dónde siempre tiene la razón y es el único que conoce y deliberadamente es quien planifica y determina la forma de habitar los moradores de los espacios, a una postura más abierta, flexible, que permita no solo la reflexión y observación del comportamiento de estos habitantes; segundo, también la participación de los habitantes en el proceso; y, tercero, la participación de otros profesionales que le permitan una visión más integrada, enriqueciendo el panorama a enfrentar, siempre con la firme convicción de mejorar la calidad de vida de los habitadores del espacio.

La arquitectura, como una estructura compleja o una construcción viva (debido a su cualidad de contemplar dentro del proceso de diseño y construcción la habitabilidad de los seres humanos habitadores), debe siempre cumplir con ciertas características que les permitan mejorar su calidad de vida y desenvolverse a través de esa variación o multiplicidad que los caracteriza al momento de habitar. Por consiguiente, esta, como dice Heidegger (1994), debe estar siempre relacionada con el pensar filosófico del arquitecto, porque el habitar y el construir son dos cosas que están íntimamente ligadas a la existencia del ser, por lo tanto, determina que el habitar es la clave de la unión, al confluir tanto el pensar como el construir, y la arquitectura debería ser la encargada de interpretar la forma de habitar de los moradores.

Como se dijo anteriormente la habitabilidad es parte de la complejidad del comportamiento humano y comprende también su relación con el entorno, las condiciones climáticas, la cultura, las tradiciones, las tecnologías, las mismas emociones humanas, así como la funcionalidad, la privacidad, la interacción con la sociedad, la economía, entre otros.

Asimismo, se puede afirmar que, la habitabilidad de los espacios arquitectónicos, son las características que estos presentan para mejorar la calidad de vida de los habitantes. No obstante, la calidad de vida siempre viene determinada por un componente psicológico propio de cada habitante. A pesar de eso, existen normas y reglamentos universales para la habitabilidad de los espacios. La habitabilidad, a pesar de ser características físicas, puede tener repercusión emocional y psicológica en los habitadores.

Por último, se destaca que, a pesar de que habitar y habitabilidad tienen significaciones diferentes, ambos se complementan para el desarrollo pleno del ser humano, ya que se vuelven fin y medio, porque el habitar representa la meta de bienestar del ser humano, pero no puede llevarse a cabo sin la relación del ser humano y su entorno (Habitar).

Por lo tanto, la importancia de que se implemente -tanto en el ejercicio de la arquitectura como en la educación (Facultades de Arquitectura) - ese pensamiento crítico de la arquitectura, basado en la correcta interpretación del comportamiento humano, así como su respeto al ambiente para generar una arquitectura que pueda trascender.

\section{Referencias}

\section{Libros}

Alexander, C. (1981) El modo intemporal de Construir. Barcelona- España, Editorial Gustavo Gili, S.A

Bauman, Z. (2006) Modernidad liquida. Buenos aires, Fondo de cultura Económica. 8va Edición.

Iglesia, R. (2010) Habitar diseñar. Buenos aires, Argentina. Ediciones de la U - Editorial Nobuko S.A.

Morín, E. (2004). Introducción al pensamiento complejo. México, Editorial Gedisa.

\section{Referencias electronicas}

Aisa, I. (2012) Arquitectura y sensibilidad. Filosofía en la arquitectura de juhani Pallasmaa. Recuperado de: https://ojs.publius.us.es/ojs/ index.php/themata/article/view/446 acceso febrero 2017.

Arcas, Pagés y Casal (2007) Habitabilidad, un concepto en crisis. Sobre su redefinición orientada hacia la sostenibilidad. Recuperado de: http://informesdelaconstruccion.revistas.csic.es/index.php/informesdelaconstruccion/article/viewFile/1271/1355 acceso en Febrero 2017. 
Sulbaran y Rangel - Importancia del Habitar en el pensamiento arquitectónico

Benavides y Hurtado. (2015) Caracterización y aportes de mejora de las condiciones de habitabilidad en asentamientos informales desde la perspectiva del desarrollo sostenible. Caso de estudio: Veraneras -Comuna 18, Santiago de Cali. Recuperado de: http://ridum. umanizales.edu.co:8080/xmlui/bitstream/handle/6789/2187/TESIS\%20CARACTERIZACI\%C3\%93N\%20VERANERAS\%20 julio\%2024.pdf?sequence=1 acceso febrero 2017.

Correa, J. (2007) La incidencia cultural, económica y del tamaño de la familia en la vivienda de interés social. Recuperado de http:// www.facartes.unal.edu.co/otros/tesis_habitat/incidencia_cultural.pdf acceso en febrero 2017.

Cuervo, J. (2009) Habitar y diseñar. El diseño como base hacia una teoría del habitar. Recuperado de: http://200.21.104.25/kepes/ downloads/Revista5_12.pdf acceso en Febrero 2017.

Delgadillo, A. (2013) Hábitat - habitabilidad la formación del arquitecto estructuración académica. Recuperado en: https:// www.researchgate.net/publication/279854013_HABITAT_-_HABITABILIDAD_LA_FORMACION_DEL_ARQUITECTO_ ESTRUCTURACION_ACADEMICA acceso en Febrero 2017.

Doberti, R. (1993) Lineamientos para una Teoría del Habitar. Recuperado en : http://webcache.googleusercontent.com/ search?q=cache:60uss_qS8ZAJ:www.fadu.uba.ar/application/post/download-filename/549+\&cd=1\&hl=es$419 \& \mathrm{ct}=\mathrm{clnk} \& \mathrm{gl}=\mathrm{ve}$ acceso en Febrero 2017.

Heidegger, M. (1956) Construir, Habitar, Pensar. Recuperado de: http://www.laeditorialvirtual.com.ar/pages/heidegger/heidegger_ construirhabitarpensar.htm acceso en febrero 2017.

Iglesia, R. ((S/F) Mimeo) Vivir y habitar. Recuperado de: https://sites.google.com/site/catedrarodriguez/ acceso en febrero 2017

López, J. (2010) La habitabilidad de la arquitectura. El caso de la vivienda. Recuperado en: http://dearq.uniandes.edu.co/sites/default/ files/articles/attachments/dearq06_08_-_Lopez_de_Asiain.pdf acceso en febrero 2017.

Martínez, E. (2014) Configuración urbana, hábitat y apropiación del espacio. Recuperado en: http://www.ub.edu/geocrit/sn/sn493/493-33.pdf acceso febrero 2017.

Mena, E. (2011) Habitabilidad de la vivienda de interés social prioritaria en el marco de la cultura. Cuadernos de vivienda y urbanismo. Recuperado en: http://revistas.javeriana.edu.co/index.php/cvyu/article/viewFile/5477/4413 acceso en febrero 2017.

Mercado, S [y otros.] (1995) Habitabilidad de la Vivienda Urbana. México: UNAM, Facultad de Psicología, Programa Universitario de Estudios sobre la Ciudad, Dirección General de Asuntos del Personal Académico.

Mues, A. (2011) Habitabilidad y desarrollo urbano sostenible. Recuperado en: http://infonavit.janium.com/janium/Documentos/035079. pdf acceso en febrero 2017.

Ramírez, A. (2012). La Habitabilidad. Recuperado de: http://www.dtic.upf.edu/ rramirez/Arponce/ LaHabitabilidad.pdf acceso marzo de 2016.

Saldarriaga, A. (1981) Habitabilidad. Bogotá, Colombia. Escala fondo editorial.

Saldarriaga, A. (2006) Habitar como fundamento de la disciplina de la arquitectura. Recuperado en: http://www.bdigital.unal.edu. co/47421/1/Revista_al_habitat.pdf acceso en febrero 2017.

Saravia. M. (2004) El significado de habitar. Recuperado en: http://habitat.aq.upm.es/boletin/n26/amsar.html acceso en febrero 2017

Sulbarán, J. (2015) Hacia una cultura arquitectónica más humana y racional. Recuperado en: http://revistas.cecar.edu.co/procesosurbanos/article/view/88 acceso en febrero 2017.

\section{Tesis doctorales}

Molero, M. (2014) La esencia del espacio Habitable y su instauración en la realidad moderna y pos moderna. Universidad del Zulia. Tesis Doctoral.

Sulbarán, J. (2017) Una visión ética de la habitabilidad. Hacia una cultura reflexiva de la arquitectura. Universidad del Zulia. Tesis doctoral 